\title{
Brucellosis serology as an alternative diagnostic test for patients with malaria-like symptoms
}

\author{
KARIN ORSEL ${ }^{1,3^{*}}$, JEREMY HO ${ }^{2}$, JENNIFER HATFIELD ${ }^{3,5}$, MANGE MANYAMA ${ }^{4}$, CARL RIBBLE ${ }^{5}$, and FRANK VAN \\ DER MEER ${ }^{3,5}$ \\ 'Department of Production Animal Health, HSC 2516, Faculty of Veterinary Medicine University of Calgary, 3330 \\ Hospital Drive NW, Calgary, AB, Canada, T2N 4N1 \\ ${ }^{2}$ Department of Community Health Sciences, TRW Building 3rd Floor, Cumming School of Medicine University of \\ Calgary, 3280 Hospital Drive NW, Calgary, AB, Canada, T2N $4 Z 6$ \\ ${ }^{3}$ Department of Community Health, TRW 7th Floor, Cumming School of Medicine University of Calgary, 3280 \\ Hospital Drive NW, Calgary AB, Canada, T2N $4 Z 6$ \\ ${ }^{4}$ Catholic University of Health and Allied Sciences, PO Box 1464, Mwanza, Tanzania \\ ${ }^{5}$ Department of Ecosystem and Public Health, HRIC 2C56, Faculty of Veterinary Medicine University of Calgary, 3330 \\ Hospital Drive NW, Calgary, AB, Canada, T2N 4N1
}

\begin{abstract}
Background: In a low transmission zone for malaria, we explored the presence of brucellosis as an alternative diagnosis in febrile patients. Specifically, the objective of our study was to determine the proportion of patients presenting to the hospital with malaria-like febrile symptoms that were concurrently brucellosis seropositive, and assess related risk factors.

Methods: The study was done at the rural hospital in Endulen, in the Ngorongoro Conservation Area (NCA), Tanzania. Hospital patients were enrolled showing malaria-like febrile symptoms and were tested for malaria (microscopy) as well as brucellosis (agglutination test, plus commercial ELISAs for IgM and for IgG). Patient demographic characteristics and risk factors for brucellosis were determined using a questionnaire interview.

Results: A total of 159 patients were enrolled, of which 57 were positive for malaria and 58 were positive by ELISA for brucellosis, with 54 (93\%) of these brucellosis-positives solely positive for isotype IgG, 1 (1.7\%) solely for isotype IgM, and $3(5.2 \%)$ positive for both IgM and IgG. However, the brucellosis agglutination test was only positive in 9 of 159 samples. Co-infections of malaria and brucellosis occurred, but malaria disease status was not significantly correlated with brucellosis status. Patients serologically positive for brucellosis were more likely to have had contact with aborted animal materials $(p=0.002)$.

Conclusions: Brucellosis is a potentially missed diagnosis amongst pastoralists in rural Tanzania with malarialike symptoms. However, concurrent and isotope-specific serology is integral to ensure detection and appropriate clinical management.
\end{abstract}

Keywords: Brucellosis, malaria, febrile, diagnosis, pastoralist, ELISA, Tanzania

\section{Introduction}

Malaria is one of the world's most important public health problems, and however the prevalence of malaria infections (including both symptomatic and asymptomatic infections) has decreased significantly in Africa, the number of people infected is still estimated to be 128 million in 2013 (WHO, 2014). It is noteworthy that clinical signs of human malaria are not pathognomonic and shared with various febrile diseases (e.g. brucellosis, typhoid, pneumonia, and other protozoal diseases). Consequently, a diagnosis based solely on clinical symptoms is unreliable, thereby highlighting the need for laboratory confirmation. Nonetheless, in areas where malaria is endemic, alternative diagnoses (e.g. brucellosis) have not consistently been considered in patients with malaria-like symptoms especially when diagnostic capacity is hampered. Other hampering factors identified

\footnotetext{
*orrespondence E-mail: karin.orsel@ucalgary.ca
} 
where awareness of the non-malaria febrile illnesses and how these illnesses are diagnosed clinically (Animut et al., 2009). Furthermore, detection of brucellosis is seriously challenged by restricted health-seeking behavior of the population at risk (Kunda et al., 2007).

Brucellosis is a global re-emerging disease, with > 500,000 new cases annually (Pappas et al., 2006). Therefore, to improve the diagnosis of brucellosis, there is a need for better understanding of the epidemiology of the disease, improved surveillance, and enhanced laboratory capacity (McDermott \& Arimi, 2002).

In northern regions of Tanzania, the seroprevalence of brucellosis in febrile patients in highrisk populations ranged from 5.5-6.2\% (Kunda et al., 2010). Due to the zoonotic nature of brucellosis, transmission risks are affected by prevalence of this organism in ruminants, and the rate and intensity of human-animal contact (McDermott \& Arimi, 2002). This is especially salient for seminomadic, pastoralist populations, who live and have daily contact with large groups of livestock (Schelling et al., 2003). Additionally, specific cultural practices, including animal slaughter for meat and blood and daily consumption of raw milk, increase the risk of infection (Kunda et al., 2010; Kang'ethe et al., 2007; Namanda et al., 2009) and co-infection with other zoonotic diseases (Bouley et al., 2012). Fortunately, ELISA-based serology facilitates identification of brucellosis-exposed patients. Where bacterial culture can confirm the clinical diagnosis, serology can identify patterns of immunoglobulin response, thereby enabling characterization of disease status as acute versus chronic (Ariza et al., 1992). High titers of detectable IgM start 1 week after infection and rise for $\sim 1$ month before reaching a plateau. Therefore, IgM is generally associated with clinically acute patients (Pabuccuoglu et al., 2011). Thereafter, IgG antibodies start to increase, however, the half-life of IgG antibodies is much longer than IgM, therefore, they are detected for up to 1 year after infection which makes the use for confirmation diagnostics more challenging. It is important to note however, that false positive ELISA results have been reported due to cross-reaction of pathogens with shared epitopes (Nielsen et al., 2010).

Brucellosis has been confirmed as a potential differential diagnosis in pastoralists presenting with flu-like symptoms in both Kenya and Ethiopia (Animut et al., 2009; Maichomo et al., 2000). Although brucellosis has been identified in Northern Tanzania (Bouley et al., 2012, Crump et al., 2013, Kunda et al., 2010) and in the Katavi-Rukwa ecosystem in the southeast (Assenga et al., 2015) however, in predominantly Maasai pastoralist populations of Tanzania and more particular in the Ngorongoro Conservation Area (NCA), seroprevalence of brucellosis amongst febrile patients suspected of having malaria has not been reported. It was noteworthy that our research group reported high rates of false-positive diagnoses of malaria at the Endulen hospital (Allen et al., 2013). Consequently, brucellosis was considered as a likely alternative diagnosis.

Therefore, the first objective of our study was to examine the proportion of brucellosis antibody-positive cases amongst patients presenting with malaria-like symptoms. Secondly, we aimed to further understand the clinical signs most likely correlated with seropositivity, and finally, to investigate if cultural practice related risk factors were associated with being serologically positive to brucellosis.

\section{Materials and methods}

\section{Study area and design}

The study was done at the rural hospital in Endulen, in the NCA, Tanzania. The hospital services a population of approximately 77,000 people in a catchment area of $8300 \mathrm{~km}^{2}$, primarily comprised of the semi-nomadic pastoralist Maasai (Allen et al., 2013). A cross-sectional study during November and December 2011 was performed. Participating medical staff assessed patients presenting to the hospital's outpatient department. Any patient that presented with a suspicion of malaria and/or 
febrile symptoms was asked to participate. During an interview questions were asked, focused on collecting information regarding patients' age, gender, duration of illness, symptoms, village of origin, consumption of raw milk, direct assistance with animal births, and possible contact with aborted animal materials. In the case of minors, parents or guardians were asked to answer these questions. Enrolled patients were then sent to the laboratory for diagnostic procedures (based on the hospital protocol), in addition to blood collection for brucellosis diagnostics.

\section{Laboratory assays}

Blood samples $(5 \mathrm{~mL})$ were collected from each participant in serum collection tubes (Vacutainer, BD, Franklin Lakes, NJ, USA) for both malaria and brucellosis diagnostics. Following the hospital protocol for malaria diagnosis, immediately after blood collection, a thick blood smear slide was prepared. Blood was centrifuged ( $2500 \mathrm{G}$ for $5 \mathrm{~min}$ ), and serum collected and aliquoted for the serum agglutination test. The remainder was stored at $-20^{\circ} \mathrm{C}$, pending shipment to the laboratory of the Catholic University Hospital of Health and Allied Sciences (CUHAS) in Mwanza for further analysis using ELISA bioassay. Frozen serum samples were packed in insulated boxes containing frozen ice packs. WarmMark ${ }^{\circledast}\left(10^{\circ} \mathrm{C}\right.$ limit) and MonitorMark ${ }^{\circledast}$ (Cold Chain Technologies, Holliston, MA, USA; $31^{\circ} \mathrm{C}$ limit) temperature monitoring strips were placed inside. Upon receipt, laboratory technicians at CUHAS assessed the integrity and condition of the serum samples, and stored them at $-40^{\circ} \mathrm{C}$ pending processing.

\section{Malaria diagnosis}

Air-dried thick blood smear slides were prepared in the hospital laboratory. They were incubated in 3\% Giemsa stain solution (Sigma-Aldrich, Oakville, ON, Canada) for approximately 30-45 min at room temperature, then washed with Giemsa buffer for $5 \mathrm{~min}$. Thereafter, slides were air-dried and examined using oil-immersion light microscopy (1000 x), as described (Allen et al., 2013).

\section{Brucellosis diagnosis}

Brucellosis agglutination tests were performed according to the manufacturer's protocol (Euromedi Equip Ltd., Edgware, UK). Briefly, 2 drops of serum were pipetted onto a slide card. One drop of (non-brucella species specific) Eurocell $\mathrm{A} / \mathrm{M}$ reagent was gently mixed with the serum using a clean, plastic stir stick. The card was rotated and rocked by hand $(5 \mathrm{~min})$. Presence or absence of agglutination was noted and recorded.

Samples were also tested using commercial ELISA assays to detect Brucella IgM or IgG (RE56821 and RE56841, respectively; Biosupply UK Ltd, Bradford, UK) according to the manufacturer's protocol. Briefly, serum samples were thawed for 4 hours at room temperature and diluted 1:100 with diluent buffer. Cutoff values were determined using controls containing 1; 10; 50 and $200 \mathrm{U} / \mathrm{mL}$ of anti-Brucella abortus IgG or IgM antibody. Using these controls, a standard curve was derived, relating Optical Densities (OD) to $\mathrm{U} / \mathrm{mL}$ of antibodies. An OD corresponding to $<8 \mathrm{U} / \mathrm{mL}$ of either $\lg G$ or $\operatorname{lgM}$ were reported as negative, between $8-12 \mathrm{U} / \mathrm{mL}$ as equivocal, and $>12 \mathrm{U} / \mathrm{mL}$ were positive. Patients were classified as either IgM or IgG positive, and a combined category was created for total positives (TotPos) if either IgM and/or IgG were positive.

\section{Data analysis}

Questionnaires were digitalized and every symptom recorded by the medical staff was noted. These reported symptoms were then grouped into various symptom categories (Table 1). 
Table 1: Recorded patient symptoms from questionnaire and related grouping symptom category

\begin{tabular}{ll}
\hline Hospital staff recorded symptoms & Symptom category (group) \\
\hline Joint pain, leg pain, bone pain, swelling of lower limbs, back & Bone and Joint Pain \\
ache, swelling of neck, waist ache, hip pain, extremities pain, & \\
difficulty walking, neck pain, shoulder pain & \\
Fever, shivering & Febrile \\
Eye ache & Ocular \\
Cough, difficulty breathing & Respiratory \\
Abnormal behavior & Psychiatric \\
Dysuria & Infectious \\
Diarrhoea, vomiting, abdominal discomfort, nausea & Gastrointestinal \\
Rashes over the body & Dermatological \\
Bleeding & Haematological \\
Chest pain, chest pain and tightness & Cardiac \\
Oral discharge & Discharge \\
\hline
\end{tabular}

Patients were counted in every symptom category where they expressed symptoms, however only counted once per symptom category. Chi-square statistics were used to examine associations between clinical symptoms and serological status for brucellosis as well as with risk factors identified in the questionnaire. For analytic purposes, missing values were only deleted for the specific fields.

\section{Ethical Considerations}

Ethical approval was provided by the University of Calgary Conjoint Health Research Ethics Board (Canada) ID\#: 23861. Ethical clearance was obtained from the National institute for Medical research (NIMR) with ref NIMR / HQ / R.8a/Vol.IX/1153. Permission to conduct this research in the Ngorongoro was also granted by the Ngorongoro Conservation Area Authority with Ref.NCAA/335/Vo.XIV/225. Medical Staff were trained to explain the objectives of the project to the patients and they sought written or verbal consent. In the case of minors, parents or guardians of minors were asked consent, before continuing with routine medical examinations. All participating medical staff members were also trained regarding the content and delivery of the interview questionnaire. Potential risks and benefits of the research were explained to each presenting patient, as well as involvement tasks and potential harms. Furthermore, patients were advised that they had the right to terminate participation at any time.

\section{Results}

\section{Socio-demographic characteristics of study subjects}

In total, 159 patients were enrolled, of which 50 (31\%) were male, $88(55 \%)$ female, and $21(13 \%)$ had no gender recorded by the staff (incomplete; missing value). Of all enrolled patients, 7 (4\%) were $<5$ years old, 37 (23\%) were between 5 and 18 years, 110 (69\%) were $>18$ years, and $5(3 \%)$ had no age data recorded by the staff (incomplete file: missing value). With only 7 patients under 5 years of age and the potential impact of maternal antibodies in young babies, we excluded these 7 patients from the risk factor analysis. All 7 young patients tested negative for brucellosis. In total, 30 villages were represented, covering a large portion of the southern NCA.

\section{Laboratory results}

Of all patients enrolled, 156 were tested for malaria, of which $36.5 \%$ ( $n=57)$ were positive based on microscopy and therefore, were classified as malaria infected. The brucellosis agglutination test classified $9(5.7 \%)$ patients as positive for Brucella spp. Of all tested sera $(n=159), 58(36.5 \%)$ were 
TotPos, and 101 (63.5\%) were negative for both IgM and IgG. Of the TotPos, 54 (93\%) were solely lgG positive, and only 1 sample (1.7\%) was solely IgM positive (Table 2 ). In addition, 3 samples were ELISApositive for both IgM and IgG. None of the symptom categories was significantly associated with brucellosis status (Table 2).

Table 2: Symptoms shown by brucellosis (Bruc) sero positive, negative and total population

\begin{tabular}{llll}
\hline & $\begin{array}{l}\text { Showing symptom in total } \\
\text { population (\%) }\end{array}$ & $\begin{array}{l}\text { Symptom in Bruc sero-pos } \\
\text { population (\%) }\end{array}$ & $\begin{array}{l}\text { Symptom in Bruc } \\
\text { sero neg } \\
\text { population (\%) }\end{array}$ \\
\hline Bone and joint & $72(45)$ & $27(47)^{\mathrm{ns}}$ & $45(45)$ \\
Febrile & $108(68)$ & $36(62)^{\mathrm{ns}}$ & $72(71)$ \\
General Body malaise & $135(85)$ & $47(81)^{\mathrm{ns}}$ & $88(87)$ \\
Gastrointestinal & $43(27)$ & $14(24)^{\mathrm{ns}}$ & $29(29)$ \\
Respiratory & $34(21)$ & $10(17)^{\mathrm{ns}}$ & $24(24)$ \\
Infectious & $10(7)$ & $5(9)^{\mathrm{ns}}$ & $5(5)$ \\
Cardiac & $55(35)$ & $20(34)^{\mathrm{ns}}$ & $35(35)$ \\
& Total $\mathrm{n}=159$ & Sero-pos $\mathrm{n}=58$ & Sero-neg $\mathrm{n}=101$ \\
\end{tabular}

ns=Not significant

Malaria parasites were detected in $24.6 \%(n=14)$ of IgG-positive patients. There were no malaria parasites detected in IgM positive patients. Malaria parasites were detected in 24 (24.0\%) of brucellosis serological-negative patients, resulting in no difference $(P=0.742)$ in being brucellosis positive or negative for having malaria parasites.

Table 3: Risk factors tested in the study population for brucellosis serology negative and positive sub populations

\begin{tabular}{lllll}
\hline Variable & Response & $\begin{array}{l}\text { Brucellosis test } \\
\text { positive (\#) }\end{array}$ & $\begin{array}{l}\text { Brucellosis test } \\
\text { negative (\#) }\end{array}$ & $\begin{array}{l}\text { Significance } \\
\text { (p-value) }\end{array}$ \\
\hline Gender & Male $(n=50)$ & 23 & 27 & 0.128 \\
Age & Female $(n=88)$ & 29 & 59 & \\
& 5 till $18(n=37)$ & 11 & 26 & 0.161 \\
Duration of sickness & $>18(n=110)$ & 47 & 63 & \\
& 0 till $7(n=65)$ & 26 & 43 & 0.485 \\
Assisting birthing of animals & $>7(n-87)$ & 30 & 57 & 0.136 \\
In contact with aborted material & Yes $(n=36)$ & 17 & 19 & \\
& No $(n=122)$ & 41 & 81 & 0.002 \\
Mes $(n=17)$ & 12 & 5 & \multirow{2}{*}{0.96} \\
& No $(n=139)$ & 45 & 94 & \\
\hline & $\operatorname{Pos}(n=38)$ & 14 & 24 &
\end{tabular}

\section{Risk factor analysis}

A greater proportion ( $\mathrm{P}=0.002)$ of brucellosis antibody-positive patients reported contact with animal abortions or aborted material than brucellosis-negative patients. There was no difference in being brucellosis antibody positive or negative for gender $(P=0.128)$ or age category $(P=0.162)$. Further, there was no difference in being brucellosis antibody positive or negative for duration of 
sickness before presenting to hospital $(P=0.485)$, consumption of non-boiled dairy products within the last month $(P=0.828)$, or directly assisting birthing of any animals $(P=0.136$; Table 3$)$.

\section{Discussion}

In our study population of patients presenting to the hospital with malaria-like symptoms, $36.5 \%$ tested positive for malaria, although similar percentages of patients tested positive for brucellosis IgG and/or IgM antibodies (as detected by ELISA). Of this test positive subpopulation, 14 patients tested positive for both diseases. The absence of any significant association between the observed clinical signs and the diagnostic outcome provided additional evidence for the inherent challenge of making a clinical diagnosis of febrile patients (Thakur et al., 2002; Dean et al., 2012). In comparable pastoralist areas and populations of Kenya, brucellosis accounted for $13 \%$ of patients with malaria-like symptoms, although confirmation was done using the Rose Bengal Test (RBT) and Serum Agglutination Test (Maichomo et al., 2000). Occupational groups at risk in Eritrea reported the highest prevalence of brucellosis (7.1\%) among dairy farm workers and owners in randomly selected dairy-cattle farms, followed by veterinary personnel and inhabitants in pastoralist areas, also based on RBT and complement fixation tests (Omer et al., 2002). Other occupationally exposed groups in northern Tanzania had an RBT seroprevalence of 5.52\% (Swai \& Schoonman, 2010). Some studies have also shown both RBT and SAT to have lower test sensitivities (Al Dahouk et al., 2003; Mantur et al., 2010; Mert et al., 2003). Other prevalence estimations also seemed lower. For example, in nonpastoralist rural Ethiopia, B. abortus infections were diagnosed in $2.6 \%$ of acute febrile patients using SAT (Animut et al., 2009). Similarly, in non-pastoralist Tanzania, 3.5\% of acute febrile patients were positive for Brucella spp. using SAT (Bouley et al., 2012). Both reported estimates were from a subgroup of the population with lower risk due to lifestyle. Health-seeking behavior in low- and middle-income countries affects prevalence estimations amongst hospital populations (Kunda et al., 2007). Therefore, sampling a brucellosis high-risk population like the pastoralist Maasai, and selecting specifically for malaria-like patients in a hospital using ELISA as a diagnostic test, may explain our higher proportions as compared to other studies.

Our cross-sectional study approach enrolled patients with illness of variable duration before presenting at the hospital, as well as variable treatment histories. Classifying them as chronic or acute patients was challenging, as this patient group presented themselves in various stages of the disease, with a variety of immunological profiles (Araj et al., 1986). Due to the health system set up, convalescent samples were not available for follow up diagnostics.

A large proportion of brucellosis antibody-positive patients also had evidence of malaria parasites, as confirmed by microscopy, which can be expected in regions where both pathogens are endemic. Brucellosis and malaria co-infections have also been reported in studies on nomadic pastoralists in northern Kenya and are further complicating febrile disease diagnosis (Ari et al., 2011). The relatively high proportion of co-infections that could be detected in the NCA, was consistent with findings in Egypt, in which a proportion of patients with undifferentiated acute febrile illnesses had laboratory evidence of combined infections, often including brucellosis (Parker et al., 2007). Consequently, strengthening access to health facilities and laboratory diagnostic capacity is imperative for improved febrile patient management. Because brucellosis and malaria are clinically indistinguishable and both present with diagnostic challenges, it will inevitably lead to malaria overdiagnosis contributing to brucellosis under-diagnosis (Franco et al., 2007). Therefore in patients with febrile symptoms from rural, pastoral environments endemic for brucellosis, systematic and combined testing for brucellosis should be considered.

Substantially more patients were positive for brucellosis based on IgG compared to IgM. These patients may be in the chronic stage of brucellosis, as IgG peaks two months after initial 
infection (Ariza et al., 1992). However, detectable IgG can also be a result of slow antibody decline after successful treatment, or may simply reflect an endemic level of antibody and not chronicity, as predominating IgG levels with low IgM are present in occupationally exposed populations (Gazapo et al., 1989). In addition to increased residual levels of antibodies, also multiple peaks in antibody titers have been described (Ariza et al., 1992). We therefore cannot conclude that Brucella spp infections explain the febrile symptoms upon presentation to the hospital.

We detected ELISA positive IgM in only four patients, of which three were also positive for IgG. These patients could have been in the acute/sub-acute stage of the disease. IgM antibodies were detected within the first two weeks after infection, before declining through negative feedback (Gazapo et al., 1989). Because IgM levels are only present during initial phases of infection, it is the most useful isotype to detect when using serology for diagnostic procedures in acutely infected patients (Araj et al., 1986). There can be an overlap during the seroconversion period where IgG concentrations rise and IgM concentrations decrease. Unfortunately, the low number of positives limited our ability to make any statistical inferences involving IgM-positive patients and is very likely a reflection of health-seeking behavior in the NCA. As is previously reported by Kunda et al. (2007) brucellosis cases showed delayed going to hospitals, with the distance to hospital, keeping animals and knowledge of the disease as significantly associated with this delay.

In the present study, contact with animal aborted material was associated with being brucellosis antibody positive across all age categories, consistent with pastoralist populations in Chad (Schelling et al., 2003). With Maasai working closely with cattle as well as sheep and goats, exposure to multiple strains of Brucella spp can be expected. Admittedly, the current study was not designed to comprehensively assess all known risk factors (based on the literature) nor did it have the power to analyze interactions between risk factors. Notwithstanding, it highlights future research opportunities related to animal-human interaction to identify important animal species and risk factors related to exposure to Brucella spp.

To reduce transmission of Brucella spp from animals to humans, a One Health approach, considering ecosystem dynamics and interactions of animal and human health, would be optimal (Zinsstag et al., 2006, 2009; Godfroid et al., 2011). In general, understanding risk factors and epidemiology through integrated surveillance, health promotion and treatment campaigns for both animal and human health are important strategies to control endemic zoonotic diseases in pastoralist populations.

In conclusion, brucellosis is a potentially missed diagnosis amongst pastoralist patients presenting with malaria-like symptoms in rural Tanzania. Clinically, brucellosis presents with symptoms indistinguishable from other febrile diseases like malaria, and highly accurate laboratory diagnostic tools like ELISA are integral for proper febrile disease management. ELISA also enables detection of the specific immunoglobulin profile of each patient. Contact with aborted animal material leads to a higher probability of being brucellosis antibody positive. Co-infection of brucellosis and malaria as observed in this study further highlights the importance of strengthening laboratory capacity for accurate diagnosis.

\section{Acknowledgements}

We acknowledge involvement of all hospital medical staff in their integral roles in ensuring the success of this research, specifically Mameso Oloishiro, Goodluck Kway, Benson Mukoya, Godwin Saningo, and Bahati Medard. It was noteworthy that the laboratory staffs, Sylvery Silvester and Rita Maloy were instrumental in data collection. We also acknowledge contributions in study design and data collection by Lisa Allen and Adam Chernick. We thank Bernard Okamo and our partners at CUHAS for facilitating ELISA analyses. We also thank the Catholic Archdiocese of Arusha Tanzania, 
and the Department of Community Health Sciences and the Faculty of Medicine University of Calgary, for their support and particular Dr. Kastelic for editing the manuscript.

\section{References}

Al Dahouk, S., Tomaso, H., Nöckler, K., Neubauer, H. \& Frangoulidis, D. (2003) Laboratory-based diagnosis of brucellosis: a review of the literature. Part 1: Techniques for direct detection and identification of Brucella spp. Clinical Laboratory 49, 487-505.

Allen, L.K., Hatfield, J.M., Manyama, M. (2013) Reducing microscopy-based malaria misdiagnosis in a low-resource area of Tanzania. Tanzania Journal of Health Research 15, 1-9.

Animut, A., Mekonnen, ET AL., Shimelis, D. \& Ephraim, E. (2009) Febrile illnesses of different etiology among outpatients in four health centers in Northwestern Ethiopia. Japanese Journal of Infectious Diseases 62, 107-110.

Araj, G.F., Lulu, A.R., Mustafa, M.ET AL. \& Khateeb, M.I. (1986) Evaluation of ELISA in the diagnosis of acute and chronic brucellosis in human beings. Journal of Hygiene 97, 457-469.

Ari, M.D., Guracha, A., Fadeel, M.A., Njuguna, C., Njenga, M.K., Kalani, R., Abdi, ET AL., Warfu, O., Omballa, V., Tetteh, C., Breiman, R.F., Pimentel, G. \& Feikin, D.R. (2011) Challenges of establishing the correct diagnosis of outbreaks of acute febrile illnesses in Africa: the case of a likely Brucella outbreak among nomadic pastoralists, northeast Kenya, March-July 2005. American Journal of Tropical Medicine and Hygiene 85, 909-912.

Ariza, J., Pellicer, T., Pallares, R., Foz, A. \& Gudiol, F. (1992) Specific antibody profile in human brucellosis. Clinical infectious diseases 14, 131-140.

Assenga, J.A., Matemba, L.E., Muller, S.K., Malakalinga, J.J. \& Kazwala, R.R. (2015) Epidemiology of Brucella infection in the human, livestock and wildlife interface in the Katavi-Rukwa ecosystem, Tanzania. BMC Veterinary Research 11, 189.

Bouley, A.J., Biggs, ET AL.M., Stoddard, R.A., Morrissey, A.B., Bartlett, J.A., Afwamba, I.A., Maro, V.P., Kinabo, G.D., Saganda, ET AL., Cleaveland, S. \& Crump, J.A. (2012) Brucellosis among hospitalized febrile patients in northern Tanzania. American Journal of Tropical Medicine and Hygiene 87, 1105-1111.

Crump J.A., Morrissey, A.B, Nicholson, W.L., Massung, R.F., Stoddard, R.A., Galloway, R.L., Ooi, E.E., Maro, V.P., Saganda, W., Kinabo, G.D., Muiruri, C. \& Bartlett, J.A. (2013) Etiology of severe non-malaria febrile illness in Northern Tanzania; a prospective cohort study. PLoS Neglected Tropical Diseases 18, 1-8.

Dean, A.S., Crump, L., Greter, ET AL., Hattendorf, J., Schelling, E. \& Zinsstag, J. (2012) Clinical manifestations of human brucellosis: a systematic review and meta-analysis. PLoS Neglected Tropical Diseases 6, e1929.

Franco, M.P., Mulder, M., Gilman, RH. \& Smits, H.L. (2007) Human brucellosis. Lancet Infectious Diseases 7, 775-786.

Gazapo, E., Gonzalez Lahoz, J., Subiza, J.L., Baquero, M., Gil, J. \& de la Concha, E.G. (1989) Changes in $\lg M$ and $\lg G$ anitbody concentrations in Brucellosis over time: importance for diagnosis and follow-up. Journal of Infectious Diseases 159, 219-225.

Godfroid, J., Scholz, H.C., Barbier, T., Nicolas, C., Wattiau, P., Fretin, D., Whatmore, A.M., Cloeckaert, A., Blasco, J.M., Moriyon, I., Saegerman, C., Muma, J.B., Al Dahouk, S., Neubauer, H. \& Letesson, J.J. (2011) Brucellosis at the animal/ecosystem/human interface at the beginning of the 21st century. Preventive Veterinary Medicine 102, 118-131.

Kang'ethe, E.K., Ekuttan, C.E., Kimani, V.N. \& Kiragu, M.W. (2007) Investigations into the prevalence of bovine brucellosis and the risk factors that predispose humans to infection among urban 
dairy and non-dairy farming households in Dagoretti Division, Nairobi, Kenya. East African Medical Journal 84, S96-100.

Kunda, J., Fitzpatrick, J., Kazwala, R., French, N.P., Shirima, G., Macmillan, A., Kambarage, D., Bronsvoort, M. \& Cleaveland, S. (2007) Health-seeking behaviour of human brucellosis cases in rural Tanzania. BMC Public Health 7, 315.

Kunda, J., Fitzpatrick, J., French, N., Kazwala, R., Kambarage, D., Mfinanga, G.S., MacMillan, A. \& Cleaveland, S. (2010) Quantifying risk factors for human brucellosis in rural northern Tanzania. PloS one 5, e9968.

Maichomo, M.ET AL., McDermott, J.J., Arimi, S.M., Gathura, P.B., Mugambi, T.J. \& Muriuki, S.M. (2000) Study of brucellosis in a pastoral community and evaluation of the usefulness of clinical signs and symptoms in differentiating it from other flu-like diseases. African Journal of Health Sciences 7, 114-119.

Mantur, B., Parande, A., Amarnath, S., Patil, G., Walvekar, R., Desai, A., Parande, M., Shinde, R., Chandrashekar, M. \& Patil, S. (2010) ELISA versus conventional methods of diagnosing endemic brucellosis. The American Journal of Tropical Medicine and Hygiene 83, 314-318.

McDermott, J.J \&, Arimi, S.M. (2002) Brucellosis in sub-Saharan Africa: epidemiology, control and impact. Veterinary Microbiology 90, 111-134.

Mert, A., Ozaras, R., Tabak, F., Bilir, M., Yilmaz, M., Kurt, C., Ongoren, S., Tanriverdi, M. \& Ozturk, R. (2003) The sensitivity and specificity of Brucella agglutination tests. Diagnostic Microbiology and Infectious Disease 46, 241-243.

Namanda, A.T., Kakai, R. \& Otsyula, M. (2009) The role of unpasteurized "hawked" milk in the transmission of brucellosis in Eldoret municipality, Kenya. Journal of Infection in Developing Countries 3, 260-266.

Nielsen, K. \& Wu, W.L. (2010) Serological diagnosis of brucellosis. Contributions Section of Biological Medical Sciences XXXI/1, 65-89

Omer, M.K., Assefaw, T., Skjerve, E., Tekleghiorghis, T. \& Woldehiwet, Z. (2002) Prevalence of antibodies to Brucella spp. and risk factors related to high-risk occupational groups in Eritrea. Epidemiology and Infection 129, 85-91.

Pabuccuoglu, O., Ecemis, T., El, S., Coskun, A., Akcali, S. \& Sanlidag, T. (2011) Evaluation of serological tests for diagnosis of brucellosis. Japanese Journal of Infectious Diseases 64, 272-276.

Pappas, G., Papadimitriou, P., Akritidis, N., Christou, L. \& Tsianos, E.V. (2006) The new global map of human brucellosis. The Lancet. Infectious Diseases 6, 91-99.

Parker, T.M., Murray, C.K., Richards, A.L., Samir, A., Ismail, T., Fadeel, M.A., Jiang, J., Wasfy, M.O. \& Pimentel, G. (2007) Concurrent infections in acute febrile illness patients in Egypt. The American Journal of Tropical Medicine and Hygiene 77, 390-392.

Schelling, E., Diguimbaye, C., Daoud, S., Nicolet, J., Boerlin, P., Tanner, M. \& Zinsstag, J. (2003) Brucellosis and Q-fever seroprevalences of nomadic pastoralists and their livestock in Chad. Preventive Veterinary Medicine 61, 279-293.

Swai, E.S. \& Schoonman, L. (2010) The use of rose bengal plate test to asses cattle exposure to Brucella infection in traditional and smallholder dairy production systems of Tanga region of Tanzania. Veterinary Medicine International pii: 837950. doi: 10.4061/2010/837950

Thakur, S.D., Kumar, R. \& Thapliyal, D.C. (2002) Human brucellosis: review of an under-diagnosed animal transmitted disease. The Journal of Communicable Diseases 34, 287-301.

WHO (2014) World Malaria Report. World Health Organization.

Zinsstag, J., Ould Taleb, M. \& Craig, P.S. (2006) Editorial: health of nomadic pastoralists: new approaches towards equity effectiveness. Tropical Medicine \& International Health : TM \& IH $11,565-568$. 
Zinsstag, J., Schelling, E., Bonfoh, B., Fooks, A.R., Kasymbekov, J., Waltner-Toews, D. \& Tanner, M. (2009) Towards a 'One Health' research and application tool box. Veterinaria Italiana 45, 121133. 